\title{
Topo-Geometric Filtration Scheme for Geometric Active Contours and Level Sets: Application to Cerebrovascular Segmentation
}

\author{
Helena Molina-Abril and Alejandro F. Frangi \\ Centre for Computational Imaging and Simulation Technologies in Biomedicine, \\ Mechanical Engineering Department, University of Sheffield, UK
}

\begin{abstract}
One of the main problems of the existing methods for the segmentation of cerebral vasculature is the appearance in the segmentation result of wrong topological artefacts such as the kissing vessels. In this paper, a new approach for the detection and correction of such errors is presented. The proposed technique combines robust topological information given by Persistent Homology with complementary geometrical information of the vascular tree. The method was evaluated on 20 images depicting cerebral arteries. Detection and correction success rates were $81.80 \%$ and $68.77 \%$, respectively.
\end{abstract}

\section{Introduction}

Accurate segmentation of the cerebral vasculature is important as part of several advanced radiological and interventional procedures; e.g. in making more objective detection of vascular pathologies (carotid stenosis, cerebral aneurysm, malformations), quantification (of stenosis grading, aneurysm measurements), personalised hemodynamic modelling, and interventional planning of endovascular procedures. Automated analysis of the cerebral vessels, however, remains challenging as it has to be robust to limited image resolution, artefacts in image acquisition, image noise, complex vascular morphologies even in healthy conditions as well as morphological alterations due to pathological conditions.

Several methods have been proposed in the literature for the segmentation of cerebral vasculature 9 . One of the main problems of the existing methods is that they are not robust to recover and preserve the topology of the underlying vascular tree. This is particularly problematic when trying to recover the full or large portions of the cerebral vasculature. Missing (e.g. due to poor image resolution or artefacts) or kissing vessels (i.e. when two distinct but parallel running vessels cannot be distinguished) can easily mislead vascular segmentation algorithms so that the final result contains missing or fused vascular segments.

In particular, due to their large presence in cerebral vasculature segmentation results, several segmentation methods address the kissing vessel problem by using shape priors that aim to prevent their formation [11813. However, these methods either require a trade-off between geometrical accuracy and topological correctness, or cannot guarantee topological correctness nor the absence

P. Golland et al. (Eds.): MICCAI 2014, Part I, LNCS 8673, pp. 755-762, 2014.

(C) Springer International Publishing Switzerland 2014 
of kissing vessels. Due to the fact that the vast majority of kissing vessels introduce topological alterations in the resulting vascular network, more general methods incorporating topological restrictions in the segmentation process could be considered a solution to the problem. Chen and Freedman [6] proposed an approach that enforces specific topological constrains within the level-set segmentation framework (called C\&F method in the sequel). This method is a general approach that allows topological control within a level set evolution by using Persistent Homology [7], which is a concept borrowed from algebraic topology. Given a known initial topology of the object to be segmented, the method is able to correct every topological error within a given segmentation. To the best of our knowledge the $\mathrm{C} \& \mathrm{~F}$ method has never been tested to quantitatively demonstrate its effectiveness on any clinical application. From our experience, the main drawback of this method is that the manipulations required for correcting the topology of the object may alter its geometry in an undesired manner leading to incorrect segmentations.

Our work builds upon the C\&F method, which is extended to enable automatic detection and semi-automatic correction of kissing vessels in cerebrovascular image segmentations. We believe that this is the first method specifically dealing with the detection and correction of such artefacts. This is particularly relevant in view of population imaging efforts where one is interested in extracting quantitative image information from large image databases, and where manual detection and correction have a huge impact in terms of reproducibility and processing time.

\section{Persistent Homology for Segmentation Errors Correction}

Given a segmentation result, the C\&F method makes use of the concepts of homology groups and persistent homology to detect and eliminate incorrect topological features that are present in the initial segmentation. This approach is based in geometric active contours (GAC) [5] that is an image-based object modelling approach based on the theory of curve/surface evolution and level set frameworks. In GAC, contours are represented implicitly using the zero-level set of a higher-dimension function called the level set function. Given a known initial topology of the object to be segmented, the C\&F method automatically drives the evolving contour within the GAC framework towards the correct object's topology. Let us introduce some of the concepts, coming from homology theory, that are used in this method.

Homology groups are efficiently computable topological invariants that have already proved their usefulness in different applications and their potential in multidimensional digital image analysis [7]. The homology groups $H(O)$ of an $n$-dimensional digital object $O$ provide information on the number of connected components and holes of various dimensions. For instance, in a $3 D$ image, 0 -holes can be seen as connected components, 1-holes as handles, and 2-holes as voids in the image. These topological features of the image are algebraically represented 
by the so-called homology classes. Intuitively, if we focus on a canonical basis of the vector space formed by the group of homology classes, we could think of having one homology class for each topological feature in the image. For example, if we think on the vessels in Fig. 1 (a) as solid tubes, there is one connected component and two handles, that is one 0-dimensional homology class and two 1-dimensional homology classes (algebraically independent). The $\mathrm{C} \& \mathrm{~F}$ method makes use of these concepts to remove the topological errors of a given segmentation. That is, knowing the correct topology of the object to be segmented, the method assures the elimination of the undesired homology classes. This elimination is performed by an automatic evolution of the object's contour that is based on its homological information. The contour of the object is implicitly represented as the zero level set $\phi: \Omega \rightarrow \mathbb{R}$ where $\Omega$ is the domain of interest and $\phi$ is the signed distance function. The method uses Persistent Homology for the homology computation of the domain $\Omega$. Persistent homology is a technique for computing topological features of a given topological space, which at the same time provides meaningful measurements of its topological properties. Given a domain $\Omega$ and a function $\phi: \Omega \rightarrow \mathbb{R}$ for each $t \in \mathbb{R}$, the persistent homology algorithm grows the sublevel set $\phi^{-1}(-\infty, t]$ from the empty set to the entire domain. Throughout this process, the algorithm detects the points (called critical points) in which homology changes (the number of $p$ dimensional algebraically independent homology classes increases or decreases).

Once the critical points are computed, and supposing that the contour we are segmenting is homeomorphic to a $d$-sphere, the C\&F method makes use of the concept of robustness [3]. Roughly speaking, given a homology class $\alpha \in H(O)$ of an object $O$, its robustness $\rho_{\phi}(\alpha)$ is a measure of how much $\phi$ needs to be modified to get rid of the topological feature represented by $\alpha$. Formally, the robustness $\rho_{\phi}(\alpha)$ is the minimal $r$ such that there exists an $r$-perturbation of $\phi$ on which $\alpha$ disappears in the perturbed object $h^{-1}(\infty, 0]$. An $r$-perturbation, $h$ of $\phi$, is the real-valued function $h$ such that $\|h-\phi\|_{\infty}=\max _{x \in \Omega}|h(x)-\phi(x)| \leq r$. The total robustness is defined as: $\operatorname{Rob}_{k}(O)=\sum_{\alpha \in H(O)}^{n} \rho_{\phi}(\alpha)^{k}-\left(\max _{\alpha \in H(O)} \rho_{\phi}(\alpha)\right)^{k}$, where $n$ is the number of homology classes in $O$. The evolution of a flow that drives the contour $C$ and its signed distance function $\phi$ towards the minimum total robustness allows the elimination of its homology classes. The final evolution equation (considering for instance degree $k=3$ robustness) is the following:

$$
\frac{\partial E}{\partial \phi}=3\left(\sum_{\alpha \in H(O), c_{\alpha} \neq c_{m i n}} \delta\left(x-c_{\alpha}\right)\right) \operatorname{sign}(\phi(x)) \phi^{2}(x)
$$

where $\delta$ is a Dirac delta function, $c_{\alpha}$ is the critical point associated to a homology class $\alpha \in H(O)$, and $c_{\text {min }}$ is the critical point associated with the most robust homology class (the only homology class we want to keep that is representing the connected component of the object). In practice, $\delta$ is implemented as a Gaussian with variance $\sigma^{2}$. The effect of this evolution is the modification of the contour within a small neighbourhood (defined by the Gaussian) of each critical point. This modification leads to the elimination of the topological feature associated to the corresponding critical point. 


\section{Methodology}

By analysing segmentation results of the cerebral vasculature, one can realize that most of the topological errors are due to kissing vessels that emerge from poor or limited image resolution. The automatic detection of these errors is not trivial, and to the best of our knowledge no effective solution is currently available for their correction. Other topological errors such as disconnected components or voids are easier to detect and correct using connected component algorithms.

Taking into consideration that the method described in Section 2 is designed for the correction of topological errors, we could contemplate its application to the automatic detection and correction of the kissing vessels in cerebral vasculature segmentations. One example of the application of the C\&F method can be seen in Fig. 1 (b)-(d) where two kissing vessels are correctly removed after applying $\mathrm{C} \& \mathrm{~F}$ (critical points are represented as black dots). However, even if the C\&F method assures a correct topology of the result, the algorithm provides a solution that is in many occasions far from the desired one. There are two main reasons for that: First, the location of critical points does not correspond in general with the location of the kissing vessel. Second, the evolution of the contour within a predefined neighbourhood (determined by the variance of the Gauss function that approximates the Dirac function in (1)) does not respect the correct geometry of the vascular tree (see Fig. 1 (c)-(e)). In Section 3.1 we propose a new method in which, by including geometrical information of the vascular tree within the C\&F method, the localization of kissing vessels is drastically improved. In Section 3.2, a correction framework that avoids the incorrect results of the type of those shown in Fig. 1(e) is proposed. The algorithm described in [4] has been used for the initial segmentations.

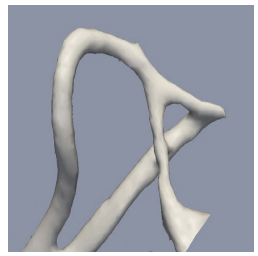

(a)

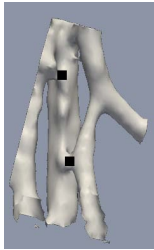

(b)

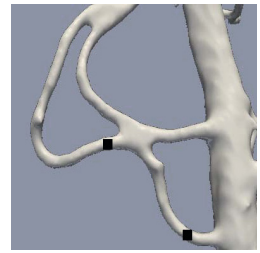

(c)

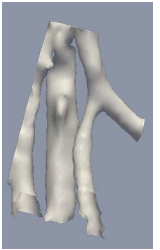

(d)

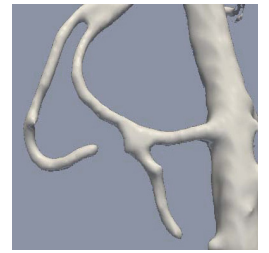

(e)

Fig. 1. (a) Part of the cerebral vasculature. (b) - (c) Initial segmentations. (d) Correct result and (e) incorrect result after applying the $\mathrm{C} \& \mathrm{~F}$ method.

\subsection{Detection Method}

Simplicial Complexes and Filtrations. A topological space for which we want to compute Persistent Homology can be represented using different structures. Simplicial complexes are convenient representations of topological spaces, specially for computer implementations, consisting of a collection of simplices that are glued together in a structured manner. A q-simplex in $\mathbb{R}^{k}$ is defined to be a set of the form: $\left\{\sum_{j=0}^{q} a_{j} v_{j}: 0 \leq a_{j} \leq 1\right.$ for $j=0, \ldots, q$ and $\left.\sum_{j=0}^{q} a_{j}=1\right\}$ 
where $v_{j}$ are affinely independent points. For example, a 0 -simplex is a vertex, a 1-simplex and edge, a 2-simplex a triangle, and a 3 -simplex a tetrahedron. Let $K$ be a simplicial complex. A filtration is a nested sequence of subcomplexes: $\emptyset=K_{0} \subset \cdots \subset K_{n}=K$. We may think of a filtration as a description of how to construct $K$ by adding blocks at a time. We can now focus in the topological evolution of a filtration of complexes by the corresponding sequence of homology groups. Since $K_{i-1} \subset K_{i}$, the inclusion map defined by $f(x)=x$ induces a homomorphism between the homology groups $f_{*}: H_{p}\left(K_{i-1}\right) \rightarrow H_{p}\left(K_{i}\right)$. The nested sequence of complexes corresponds to sequences of homology groups connected by homomorphisms, $0=H_{p}\left(K_{0}\right) \rightarrow H_{p}\left(K_{1}\right) \rightarrow \cdots \rightarrow H_{p}\left(K_{n}\right)=H_{p}(K)$, one for each dimension $p$. Given a filtration, the persistent homology algorithm considers this last sequence and detects when a new homology class is born and when an existing class dies as we proceed forward through the filtration [7. Therefore, the definition of the filter function $\phi: K \rightarrow \mathbb{R}$, is crucial within this method. If there are two simplexes whose inclusion creates a new homology class, the one that is first introduced in the filtration will be selected as critical point. In the $\mathrm{C} \& \mathrm{~F}$ method, the filter function is defined by the signed distance function. The main idea of the work presented here consists of obtaining additional geometrical information that could guide the definition of a more appropriate filtration scheme. Using this information, we create a geometry-driven filtration that gives priority to points that belong to kissing vessels to be selected as critical points.

Geometrical Properties of Kissing Vessels. The first step for incorporating geometrical information is to compute the skeleton of the segmented vasculature. We use the algorithm presented in [2] for this purpose. The skeleton of a vascular tree can be seen as a graph where the centerlines are edges and their intersections are the vertices of the graph. The geometrical properties of the kissing vessels are based on the following observations (see Fig. 2(a)-(d)): (1) They correspond with short centerlines in the vasculature skeleton, (2) its corresponding centerline are often perpendicular to its neighbouring centerlines and (3) they often cause drastic changes in the vessel radius with respect to its neighbouring centerlines. Taking into account these observations, we define a kissingness measure for each centerline $c$ of the skeleton: $K S(c)=(\pi(c)+\alpha(c)) / l(c)$, where: $(1) \pi(c)=$ $\frac{1}{n} \sum_{i=1}^{n}\left(r_{c}-r_{i}\right)^{2}$ depends on the radius of $c$ and its neighbouring centerlines. $r_{c}$ is the normalized radius of $c$ and $r_{i}$ the normalized radius of its $n$ neighbouring centerlines, for $i \in\{1 \ldots n\},(2) \alpha(c)=\frac{1}{n} \sum_{i=1}^{n} \sin (\theta(i, c))$ depends on the angle of $c$ with its neighbouring centerlines, where $i \in\{1 \ldots n\}$ are the neighbouring centerlines of $c$ and $\theta(i, c)$ is the minimum angle between the centerlines $i$ and $c$, and $(3) l(c)$ is the normalized length of $c$. The set of edges with maximal kissingness value is defined as the suspicious kissing set.

Geometry-Driven Filtration. As mentioned before, our aim is to give priority in the filtration process to the points that are close to the edges that belong to kissing vessels. Using the set of suspicious kissing edges, the idea consists in decreasing the values of the signed distance function around them, and then use this modified signed distance function as the filter function for the persistent 
homology algorithm. Having smaller filter values around the suspicious kissing edges, makes the points around them more probable to be selected as critical points (because they will be considered earlier in the persistent homology algorithm). For each suspicious kissing edge, we define a spring force 12 that acts to pull the contour toward the kissing edge middle point. Then the level set function of the initial segmentation is evolved under the influence of these forces (Fig. 2 (c)-(e)). This evolution is performed using the topological control evolution presented in [10], which guarantees the preservation of the initial contour's topology. Once this modification is done, the persistent homology of the domain delineated by the resulting contour can be computed following the filtration defined by the modified signed distance function. The set of critical points obtained after this computation are the points detected as kissing vessels.

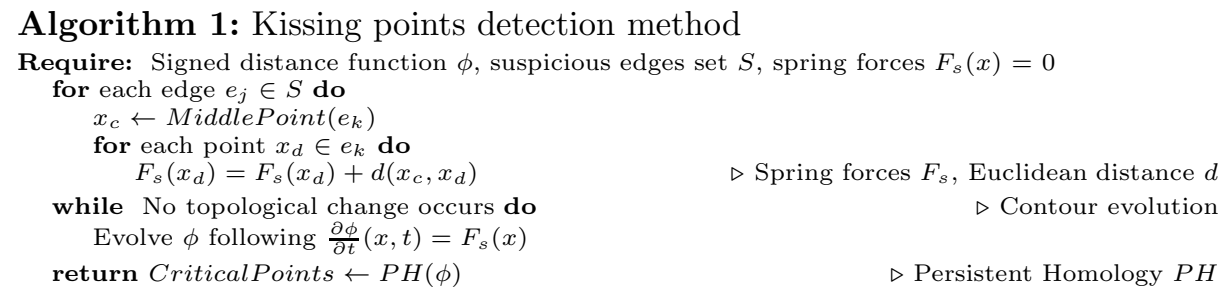

\subsection{Correction Framework}

Once the kissing vessels are detected, we could correct the topological errors in the mesh by minimizing the energy term in Equation (1). However, the correction result depends on the width of the approximation of the Dirac delta function, that is the neighbourhood of the critical point on which the evolution will have effect. Due to the wide variety of kissing vessel's shapes and locations, their automatic correction leads to geometrically incorrect results (Fig. 2 (f)-(h)). We propose here a semi-automatic correction framework in which the user can iteratively modify the segmentation results via a simple, easy-to-use graphical interface. Once the critical points have been automatically detected, instead of using a predefined neighbourhood (as in $\mathrm{C} \& \mathrm{~F}$ ), we use adjustable ellipsoids to define the neighbourhood for Equation (1). For each critical point, we create an ellipsoid centred at it. The framework allows the user to modify its axes, position and inclination. After the user has finished adjusting it to the concrete kissing vessel, the method automatically eliminates the kissing vessel applying Equation (1) within the neighbourhood defined by the ellipsoid (Fig. 2 (i)-(j)).

\section{Results}

The proposed method was compared with the C\&F method using twenty patients scanned with 3D rotational X-ray angiography. Diagnostic images were acquired as part of the @neurIST project [1. The persistent homology algorithm that is core to the $\mathrm{C} \& \mathrm{~F}$ method requires $O\left(\mathrm{~m}^{3}\right)$ operations in the worst 
(a)

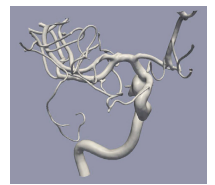

(e)

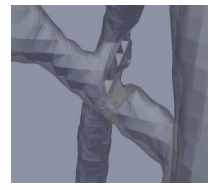

(f)

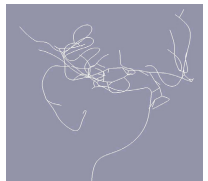

(b)

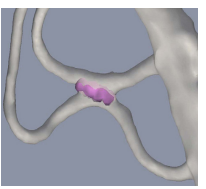

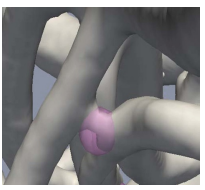

(j)

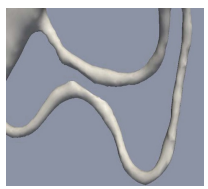

(c)
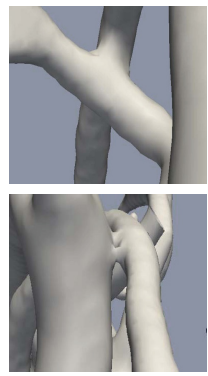

(d)

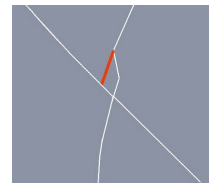

(h)

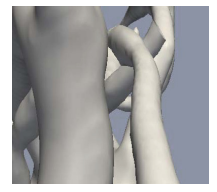

$(\mathrm{k})$

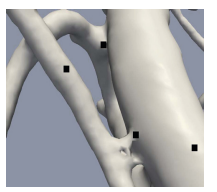

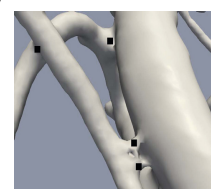

Fig. 2. (a) Initial segmentation and (b) its skeleton. (c) Kissing vessel (d) its skeleton (the red centerline is suspicious) and (e) the signed distance function after the spring forces evolution. (f) The influence of the Gaussian for $\sigma^{2}=0.4$. (g) Segmentation result and (h) correction result for $\sigma^{2}=0.1$. (i) Ellipsoidal neighbourhood. (j) Correction result. (k) Points detected with the $\mathrm{C} \& \mathrm{~F}$ method and (l) with the proposed method.
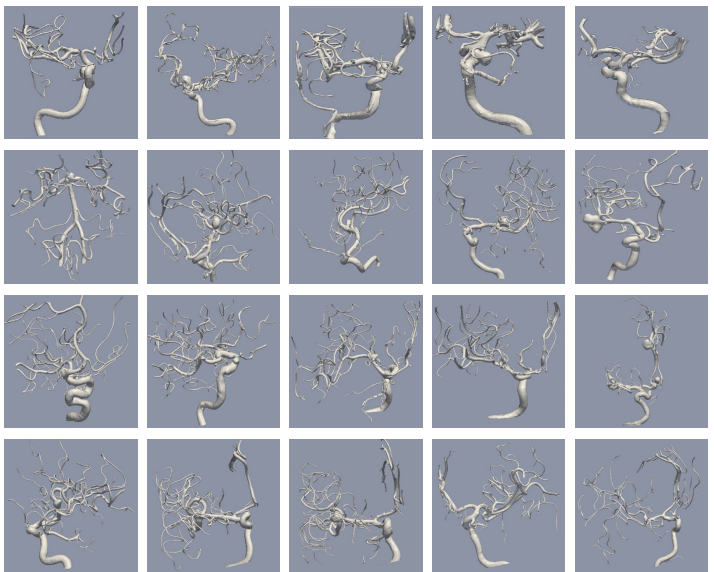

\begin{tabular}{|c|c|c|c|c|c|c|}
\hline & \multicolumn{2}{|c|}{ Detection } & Correction & \\
\hline & C\&F & Proposed & C\&F & Proposed & \#kissing \\
\hline 1 & 65.21 & 95.65 & 0.13 & 65.21 & 23 \\
2 & 48.83 & 76.74 & 25.58 & 72.09 & 43 \\
3 & 44.82 & 65.51 & 0.00 & 44.82 & 29 \\
4 & 56.52 & 95.65 & 17.39 & 95.65 & 23 \\
5 & 53.85 & 84.61 & 19.23 & 84.61 & 26 \\
6 & 40.00 & 85.00 & 10.00 & 75.00 & 20 \\
7 & 43.24 & 75.67 & 5.40 & 64.86 & 37 \\
8 & 48.57 & 82.85 & 8.57 & 68.57 & 35 \\
9 & 42.85 & 92.85 & 0.00 & 78.57 & 14 \\
10 & 50.00 & 83.33 & 8.33 & 66.67 & 12 \\
11 & 42.85 & 100.00 & 0.00 & 100.00 & 15 \\
12 & 39.13 & 86.95 & 0.00 & 65.21 & 23 \\
13 & 38.00 & 63.00 & 0.00 & 50.00 & 8 \\
14 & 38.46 & 62.00 & 7.69 & 46.15 & 13 \\
15 & 36.36 & 72.72 & 9.09 & 55.00 & 11 \\
16 & 46.66 & 83.33 & 6.66 & 56.66 & 30 \\
17 & 40.74 & 77.77 & 0.11 & 62.96 & 27 \\
18 & 44.10 & 73.68 & 15.78 & 73.68 & 19 \\
19 & 28.57 & 85.71 & 0.00 & 78.57 & 14 \\
20 & 25.00 & 92.85 & 0.00 & 71.42 & 14 \\
\hline Total & 42.92 & $\mathbf{8 1 . 8 0}$ & 6.70 & $\mathbf{6 8 . 7 7}$ & 426 \\
\hline
\end{tabular}

Fig. 3. Segmentation results. Detection, correction rates and number of initial kissings.

case, where $m$ is the number of simplices. The inclusion of the geometrical filtration takes $O(m)$ and hence introduces negligible extra computational cost. The initial segmentations and the percentage of correctly located kissing vessels for both methods are shown in Fig. 3. The rate increases from $42.92 \%$ with the C\&F method to $81.80 \%$ with our approach. An example is shown in Fig. 2 (k)-(l), where our method allowed a correct location of every kissing vessel and using the $\mathrm{C} \& \mathrm{~F}$ method only two out of four were correctly located. With respect to the correction method, we run the $\mathrm{C} \& \mathrm{~F}$ algorithm for three different values 
of $\sigma=0.1,0.5,0.9$. Best results were obtained for $\sigma=0.5$ with only $6.7 \%$ of the kissing vessels appropriately removed. We increased the correction rate to $68.77 \%$. The average number of kissing vessels per image is 21.8 . This number gives an idea of how tedious manual correction of such artefacts could be in contrast with the proposed automatic detection and semi-automatic correction.

\section{Conclusions}

We propose a new approach, combining topological and geometrical information of the vascular tree, which is able to detect in a high percentage of cases the kissing vessel errors. The proposed approach outperforms the state-of-the-art methods dealing with topological noise removal. A semi-automatic framework for the correction of such errors is also introduced, significantly reducing the user interaction time with respect to manual correction.

\section{References}

1. Aneurist project, http://www.aneurist.org

2. Antiga, L.: Patient-Specific Modeling of Geometry and Blood Flow in Large Arteries. Ph.D. thesis, Politecnico di Milano (2002)

3. Bendich, P., Edelsbrunner, H., Kerber, M.: Computing robustness and persistence for images. IEEE Trans. on Vis. and Comp. Graphics 16(6), 1251-1260 (2010)

4. Bogunović, H., Pozo, J.M., Villa-Uriol, M.C., Majoie, C.B.L.M., van den Berg, R., Gratama van Andel, H.A.F., Macho, J.M., Blasco, J., San Román, L., Frangi, A.F.: Automated segmentation of cerebral vasculature with aneurysms in 3DRA and TOF-MRA using geodesic active regions. Med. Phys. 38(1), 210-222 (2011)

5. Caselles, V.: Geometric models for active contours. In: International Conference on Image Processing. vol. 3, pp. 9-12. IEEE (1995)

6. Chen, C., Freedman, D.: Topology noise removal for curve and surface evolution. In: Menze, B., Langs, G., Tu, Z., Criminisi, A. (eds.) MICCAI 2010. LNCS, vol. 6533, pp. 31-42. Springer, Heidelberg (2011)

7. Edelsbrunner, H., Harer, J.: Computational Topology. Amer. Math. Soc. (2010)

8. Friman, O., Hindennach, M., Kühnel, C., Peitgen, H.O.: Multiple hypothesis template tracking of 3D vessel structures. Med. Imag. Anal. 14(2), 160-171 (2010)

9. Lesage, D., Angelini, E.D., Bloch, I., Funka-Lea, G.: A review of 3D vessel lumen segmentation techniques. Med. Imag. Anal. 13(6), 819-845 (2009)

10. Ségonne, F.: Active contours under topology control genus preserving level sets. International Journal of Computer Vision 79(2), 107-117 (2008)

11. Wong, W.C.K., Chung, A.C.S.: Probabilistic vessel axis tracing and its application to vessel segmentation with stream surfaces and minimum cost paths. Med. Imag. Anal. 11(6), 567-587 (2007)

12. Xu, C., Pham, D.L., Prince, J.L.: Image segmentation using deformable models. In: Handbook of Medical Imaging. Medical Image Processing and Analysis, vol. 2, pp. 175-272 (2000)

13. Yureidini, A., Kerrien, E., Cotin, S.: Robust RANSAC-based blood vessel segmentation. In: SPIE Medical Imaging, vol. 8314, p. 8314M. SPIE Press (2012) 